\title{
A influência do ambiente urbano na escolha do transporte ativo e sua relação com o sedentarismo: reflexões sobre o caso de Belo Horizonte (MG)
}

\section{Janaina Amorim Dias}

Metrics Mobilidade, Brasil.

ORCID: https://orcid.org/o0oo-0002-3155-4618

\section{Leandro Cardoso}

Departamento de Engenharia de Transportes e Geotecnia, Escola de Engenharia, UFMG,

Brasil.

ORCID: https://orcid.org/oooo-0oo1-7133-5033

\section{Leise Kelli de Oliveira}

Departamento de Engenharia de Transportes e Geotecnia, Escola de Engenharia, UFMG,

Brasil.

ORCID: https://orcid.org/oooo-0002-4756-4183

\section{Bárbara Abreu Matos}

Departamento de Engenharia Urbana, Escola de Minas, UFOP, Brasil.

ORCID: https://orcid.org/00oo-0002-0814-8195

\section{Daniela Antunes Lessa \\ Departamento de Engenharia Civil, Escola de Minas, UFOP, Brasil. \\ ORCID: https://orcid.org/oooo-0002-4856-5671}

\section{Marconi Gomes da Silva}

SPORTIF - Clínica do Exercício e do Esporte, Brasil.

ORCID: https://orcid.org/oooo-0001-6514-7183

\section{Resumo}

Neste artigo é analisada a influência do ambiente urbano na escolha do transporte ativo e nos níveis de sedentarismo em Belo Horizonte. Para tanto, a regressão logística binária foi utilizada na identificação das variáveis que influenciam a escolha entre os 
modos ativos e o automóvel. Os resultados indicam a maior probabilidade de pessoas com maiores Índices de Massa Corporal optarem pelo automóvel. Para os respondentes, a topografia e a distância são variáveis que influenciam nessa escolha. Ainda, a chance de utilização do transporte ativo diminui à medida que a renda aumenta e os níveis de sedentarismo são menores naqueles que utilizam esse modo. Por fim, neste estudo é sugerido que o ambiente urbano influencia a escolha pelo transporte ativo que, consequentemente, influencia os índices de sedentarismo. Essa compreensão é um importante instrumento para o planejamento e gestão da mobilidade, à luz da configuração e desenho do espaço urbano e da saúde pública.

Palavras-chave: Mobilidade urbana. Mobilidade ativa. Sedentarismo.

\title{
The influence of the urban environment on the choice of active transport and the relationship with sedentarism: reflections on the case of Belo Horizonte (MG)
}

\begin{abstract}
In this paper, the influence of the urban environment on the choice of active transport and sedentary levels in Belo Horizonte is analyzed. To this end, binary logistic regression was used to identify the variables that influence the choice between active modes and automobile. The results indicate that people with higher Body Mass Index are more likely to choose the car. For respondents, topography and distance are variables that influence this choice. In addition, the chance of using active transport decreases with income increases and the levels of physical inactivity are lower in those who use this mode. Finally, in this paper, it is suggested that the urban environment influences the choice for active transport, which consequently influences the inactivity rates. In this context, these reflections are an important tool for urban planning and the management of mobility regarding the structure and the design of urban space and public health.
\end{abstract}

Keywords: Urban mobility. Active mobility. Sedentarism.

Palabras clave: Movilidad urbana. Movilidad activa. Sedentarismo.

\section{Introdução}

A existência de condições adequadas para a mobilidade das pessoas é um fator determinante para que as cidades cumpram o seu papel na viabilização de relações de troca de bens e serviços, cultura e conhecimento entre os cidadãos. O surgimento do automóvel, a sua popularização e modernização (também associadas a outros modos motorizados individuais de transporte) trouxeram muitos aspectos positivos no desenvolvimento socioeconômico das cidades. Entretanto, em contraponto aos benefícios gerados pelo uso ampliado do automóvel, a somatória do aumento da capacidade de acesso e consumo de carros e motocicletas, com a escalada de investimentos em infraestruturas rodoviárias, auxiliaram na remodelagem dos centros urbanos, contribuindo para o crescimento horizontal das cidades. Sendo assim, a predominância do uso do automóvel contribuiu para a expansão do território urbano, que se tornou cada vez mais espraiado, para o aumento dos congestionamentos viários e da poluição do ar, retirando vitalidade urbana das regiões centrais das cidades, além de ocasionar uma desumanização geral dos centros urbanos (Vieira et al., 2016). Além disso, as cidades brasileiras vêm apresentando uma série de dificuldades na gestão da mobilidade urbana. Paralelamente, os índices de sedentarismo e doenças crônicas vêm aumentando, com consequente piora nas condições de saúde da população. 
Esses cenários podem estar relacionados, uma vez que é comum observar a alegação de falta de tempo para a realização de exercícios físicos e, consequentemente, de adoção de cuidados com a saúde. Cada vez mais, as pessoas deixam de se movimentar e, devido ao uso excessivo dos automóveis, via de regra, não associam a realização de atividades físicas como parte dos deslocamentos urbanos cotidianos. Nesse sentido, aponta-se por uma oportunidade para estimular o transporte ativo que, ao mesmo tempo, auxilia na melhoria da mobilidade urbana e do próprio ambiente urbano e contribui para a promoção da saúde das pessoas, ao torná-las fisicamente mais ativas.

A conveniência do transporte motorizado reduz, ainda, a dependência física para trajetos mais extensos ou com maior altimetria, propiciando ainda um aumento do comportamento sedentário, ao favorecer deslocamentos denominados porta a porta (González-Gross et al., 2013). Como consequência, tem-se o aumento da obesidade, que reduz a qualidade e a expectativa de vida das pessoas. No Brasil, a proporção de beneficiários de planos de saúde com excesso de peso vem aumentando desde 2008, tendo passado de 46,5\% em 2008 para 52,3\% em 2015 (CNT e NTU, 2017). De maneira similar, a proporção de obesos aumentou de $12,5 \%$ para $17,0 \%$ no mesmo período (Brasil, 2017). Dessa maneira, a mobilidade ativa pode ser mais um instrumento no auxílio da redução dos níveis de sedentarismo e, consequentemente, de obesidade dos residentes nas cidades brasileiras.

Belo Horizonte, capital de Minas Gerais, afigura-se como um exemplo esclarecedor desses processos, pois, segundo o Balanço Anual da Mobilidade Urbana de Belo Horizonte 2019 (Ano-base, 2018), entre 2002 e 2012, a porcentagem de viagens por automóveis aumentou de $25 \%$ para $32,6 \%$, o percentual de viagens em modos coletivos diminuiu de $44,6 \%$ para $28,1 \%$, a mobilidade a pé aumentou de $28,5 \%$ para $34,8 \%$ e o transporte por bicicleta diminuiu de $0,7 \%$ para $0,4 \%$ do total de viagens. Adicionalmente, dados do Ministério da Saúde mostram que $64 \%$ da população belo-horizontina demonstra comportamento sedentário (Brasil, 2012). Com essa realidade da cidade, na qual grande parte da população privilegia o transporte particular motorizado e apresente comportamento sedentário, pode-se sugerir que Belo Horizonte, caso faça investimentos em infraestruturas e campanhas e ações educacionais que incentivem as alternativas ativas para mobilidade urbana, poderá estimular a mudança no comportamento de parte dessa população insuficientemente ativa e inativa, promovendo, assim, a melhoria dos indicadores de saúde, como menor incidência de doenças crônicas não transmissíveis, mortalidade por todas as causas e mortalidade por doenças cardiovasculares.

Diante desse contexto, emergem as seguintes questões de pesquisa: como o ambiente urbano influencia na escolha de um determinado modo de transporte? Como o modo de transporte influencia no sedentarismo? Nesse sentido, o objetivo deste artigo é analisar a influência do ambiente urbano na escolha do transporte ativo como forma de deslocamento e as suas relações com a saúde das pessoas. Para tanto, a partir de dados de uma pesquisa realizada com residentes de Belo Horizonte, identificou-se a percepção dos respondentes em relação à influência dos fatores do ambiente urbano na escolha do transporte ativo e como o modo de transporte e as variáveis socioeconômicas podem influenciar no sedentarismo. A partir dos resultados deste estudo, pretende-se lançar luz à dimensão do impacto da mobilidade urbana ativa na saúde pública.

A mobilidade ativa faz parte de uma política sustentável e saudável, apresentando diversas vantagens como baixo custo e grandes impactos positivos em relação ao meio ambiente, quando comparados aos transportes motorizados, sobretudo por não consumir fontes de energia não renováveis, como combustíveis fósseis (Silva, 2015). Em linhas gerais, os estudos sobre os modos ativos buscam compreender, principalmente, os parâmetros relacionados ao ambiente natural e construído que influenciam o seu uso, 
como apresentado por Vieira et al. (2016). Outros refletem sobre as condições mobilidade e sua relação com o planejamento urbano (Giles-Corti et al., 2016), a qualidade de vida (Machado, 2010) e com os crescentes níveis de sedentarismo observados nas populações urbanas (Bell et al., 2002; Reynolds et al., 2010 e Scheepers et al., 2014). Nesse sentido, este artigo traz significativas contribuições para o campo de pesquisa, ao considerar as condicionantes de escolha pelos modos ativos a partir da perspectiva da população, relacionando as condições de mobilidade e o ambiente urbano com os impactos na saúde pública.

\section{A importância da mobilidade ativa para a saúde}

A dinâmica urbana atual exige tempo, recursos e disciplina para que uma pessoa estabeleça uma rotina de atividades físicas. Nesse contexto, o transporte ativo se apresenta como alternativa viável para a inclusão de atividades físicas na vida das pessoas, principalmente obesos, sedentários e idosos, por ser uma prática que tem o potencial de estimular os indivíduos a começarem a realizar práticas esportivas, dentre outras atividades que combatem o sedentarismo. Estudos atestam que pessoas que são mais ativas têm demonstrado elevado índice de satisfação, menos estresse, maior sensação de relaxamento e maior sensação de liberdade (Anable e Gatersleben, 2005; Lajeunesse e Rodríguez, 2012; St-Louis et al., 2014).

Para um adulto ser considerado fisicamente ativo, a Organização Mundial da Saúde (OMS) recomenda a realização de, pelo menos, 150 minutos (2,5 horas) semanais de exercícios físicos de intensidade moderada. Dados recentes mostram que 100 minutos de ciclismo urbano ou 170 minutos de caminhada também atendem a tal recomendação (WHO, 2017). Contudo, $49 \%$ da população brasileira está insuficientemente ativa e $15 \%$ foi considerada inativa, ou seja, não faz exercícios físicos na intensidade recomendada (CNT e NTU, 2017). Ainda, 64\% da população brasileira não pratica exercícios físicos na intensidade e volume recomendados pela OMS (CNT e NTU, 2017). Esta não é uma realidade apenas brasileira, visto que os problemas de mobilidade urbana são recorrentes em várias cidades do mundo e o comportamento sedentário atinge a maioria da população urbana mundial, gerando consequências em vários aspectos relacionados à saúde.

Nesse contexto, Bell et al. (2002) associam a posse de um veículo particular à obesidade em homens e mulheres. Esta, por sua vez, perde apenas para o tabagismo como fator contribuinte para a mortalidade (Bell et al., 2002). Assim como a obesidade, o sedentarismo está associado ao aumento da mortalidade, da ocorrência de doenças cardiovasculares, diabetes tipo 2, câncer e danos à saúde mental (Washington, 2018).

Scheepers et al. (2014) asseguram que o incentivo à mobilidade ativa proporciona efeitos positivos para a saúde das pessoas. A adoção dos modos ativos de mobilidade urbana pode contribuir para a melhoria nos indicadores de saúde, a partir da redução dos níveis de sedentarismo, dos problemas respiratórios, devido à diminuição da poluição atmosférica, da redução do risco de doenças cardiovasculares, propiciando benefícios evidentes na melhoria da saúde física e mental, e aumento do bem-estar. Contudo, ainda são necessários esforços na obtenção dados de alta qualidade para criar estatísticas mais assertivas a este respeito (Scheepers et al., 2014).

Segundo Reynolds et al. (2010), a caminhada e o ciclismo são meios de transporte promissores para reverter os níveis de inatividade da população, ocasionando um impacto satisfatório à sua saúde. Ademais, esses meios de transporte podem reduzir a poluição do ar e sonora provenientes do tráfego; reduzir os custos com cuidados 
de saúde e de transporte; assim como estimular um desenho urbano mais conectado e acessível (Reynolds et al., 2010). Mesmo diante de tantos benefícios, um estudo realizado no Brasil demonstrou que apenas $33,4 \%$ dos adultos e $26,1 \%$ da população idosa realizam deslocamento ativo (150 minutos/semana), constatando que andar de bicicleta ou caminhar como forma de deslocamento ainda é incomum no país, com poucas repercussões positivas na saúde coletiva (Madeira, 2013).

Para Gehl e Svarre (2017), o que se busca, atualmente, é ter cidades vivazes, seguras, saudáveis e sustentáveis, e "o desejo de se ter uma cidade viva é reforçado quando as pessoas são convidadas a andar, a pedalar e a "estar" em seu espaço" (Gehl e Svarre, 2017 , p. 14). Nesse contexto, o desenho urbano e as políticas de planejamento urbano têm a capacidade de moldar a saúde e o bem-estar das pessoas, além de influenciarem questões sobre as desigualdades sociais e de saúde (Villanueva et al., 2015). Dessa forma, as políticas e regulamentos de planejamento urbano e de transporte podem influenciar a localização de destinos essenciais das atividades diárias das pessoas (como lojas, ambientes de trabalho e estudo, locais de recreação e socialização) e a facilidade com que esses espaços podem ser alcançados por meio da mobilidade ativa e do uso do transporte público (Giles-Corti et al., 2016).

\section{Metodologia}

Neste estudo, os dados são provenientes de um questionário, que teve como objetivo obter informações sobre as condições de saúde e o perfil de deslocamentos cotidianos dos entrevistados, bem como a percepção destes sobre o uso de modos ativos na cidade de Belo Horizonte. O quadro 1 apresenta a estrutura do questionário, que tem três principais blocos: (i) dados socioeconômicos e de saúde, (ii) fatores do ambiente urbano que influenciam no deslocamento por caminhada e, (iii) fatores do ambiente urbano que influenciam no deslocamento por bicicleta. Usando redes sociais para a sua divulgação, a pesquisa foi compartilhada entre os residentes da cidade de Belo Horizonte, maiores de 18 anos.

Quadro 1. Estrutura do questionário. Fonte: Elaboração própria.

\begin{tabular}{lll}
\hline Bloco & Variável & Tipo de resposta \\
\hline \begin{tabular}{l} 
(i) Dados $\begin{array}{l}\text { socioeconômicos } \\
\text { de saúde }\end{array}$ \\
\cline { 2 - 3 }
\end{tabular} & Gênero & Binária \\
\cline { 2 - 3 } & Idade & Categórica (6 classes) \\
\cline { 2 - 3 } & Peso & Contínua \\
\hline & Bairura & Contínua \\
\hline & Escolaridade & Categórica (487 classes) \\
\cline { 2 - 3 } & Motivo do deslocamento diário principal & Categórica (5 classes) \\
\cline { 2 - 3 } & Modo de principal deslocamento diário & Categórica (8 classes) \\
\cline { 2 - 3 } & Fumante regular & Binária \\
\cline { 2 - 3 } & Quantidade de atividade física semanal & Categórica (3 classes) \\
\hline
\end{tabular}




\begin{tabular}{|c|c|c|}
\hline Bloco & Variável & Tipo de resposta \\
\hline \multirow{11}{*}{$\begin{array}{l}\text { (ii) Fatores do } \\
\text { ambiente urbano } \\
\text { que influenciam no } \\
\text { deslocamento por } \\
\text { caminhada }\end{array}$} & Falta de iluminação pública à noite & \multirow[t]{11}{*}{ Categórica (4 classes) } \\
\hline & Falta de vestiários nos edifícios & \\
\hline & Velocidade dos veículos nas vias & \\
\hline & Falta de segurança no trânsito (acidentes) & \\
\hline & Desconforto gerado por assédio & \\
\hline & Falta de segurança pública (assaltos) & \\
\hline & Falta de arborização (sombra) & \\
\hline & Ruído dos veículos & \\
\hline & Largura das calçadas & \\
\hline & Condição das calçadas & \\
\hline & Topografia (inclinação) & \\
\hline \multirow{9}{*}{$\begin{array}{l}\text { (iii) Fatores do } \\
\text { ambiente urbano } \\
\text { que influenciam no } \\
\text { deslocamento por } \\
\text { bicicleta }\end{array}$} & Falta de integração com transporte público & \multirow[t]{9}{*}{ Categórica (4 classes) } \\
\hline & Falta de segurança nas vias & \\
\hline & Desconforto gerado por assédio & \\
\hline & Falta de segurança pública (assaltos) & \\
\hline & Falta de bicicletários & \\
\hline & Falta de vestiários nos edifícios & \\
\hline & Falta de infraestrutura viária & \\
\hline & Velocidade alta das vias & \\
\hline & Topografia (inclinação) & \\
\hline
\end{tabular}

Os dados do bloco (i) permitiram caracterizar o respondente em relação aos deslocamentos diários e a saúde. Os dados de peso e altura foram utilizados para calcular o Índice de Massa Corporal (IMC), medida tradicionalmente utilizada para avaliação da adiposidade corporal, sendo calculado pela divisão entre a massa corporal (em quilos) e a altura (em metros) elevada ao quadrado $\left(\mathrm{kg} / \mathrm{m}^{2}\right)$. Mesmo não sendo totalmente correlacionado com a gordura corporal, é considerado um bom indicador, por se tratar de uma medida simples, prática e sem custos (Abeso, 2016). O IMC também pode ser associado às doenças crônicas não transmissíveis (DCNT) ou mortalidade. A classificação adaptada pela OMS (Quadro 2) baseia-se em padrões internacionais desenvolvidos para pessoas adultas e descendentes de europeus. Valores acima de 25 indicam sobrepeso ou obesidade.

Quadro 2. Índice de Massa Corporal e risco de doenças. Fonte: WHO (2006).

\begin{tabular}{llcc}
\hline IMC $\left(\mathrm{kg} / \mathrm{m}^{2}\right)$ & Classificação & Obesidade Grau/Classe & Risco de Doença \\
\hline$<18,5$ & Magro ou baixo peso & 0 & Normal ou elevado \\
\hline $18,5-24,9$ & Normal ou eutrófico & 0 & Normal \\
\hline $25-29,9$ & Sobrepeso ou pré-obeso & 0 & Pouco elevado \\
\hline $30-34,9$ & Obesidade & I & Elevado \\
\hline $35-39,9$ & Obesidade & II & Muito elevado \\
\hline$\geq 40$ & Obesidade grave & III & Muitíssimo elevado \\
\hline
\end{tabular}

Ainda, com o intuito de identificar se o automóvel contribui para o sedentarismo, foi utilizada a Análise de Variância (ANOVA), verificando se há diferença significativa 
entre os grupos de comportamento sedentário (inativos e insuficientemente ativos) e ativo e o IMC, para os respondentes usuários do transporte privado motorizado. Adicionalmente, realizou-se Teste de Bonferroni para identificar as variáveis com médias diferentes como forma de validar os resultados obtidos na Análise de Variância.

Por fim, um modelo de regressão ordenado foi estimado para identificar quais fatores influenciam no sedentarismo. Usando o pacote RChoice (Sarrias, 2016) na linguagem R, considerou-se o sedentarismo como variável resposta e as variáveis gênero, idade, renda, modo de transporte e IMC como variáveis explicativas. Uma variável tem influência no sedentarismo se o p-valor do seu coeficiente é estatisticamente significativo, isto é, p-valor $<0,05$.

\section{Resultados E Discussões}

\section{Amostra}

A partir da pesquisa online foram obtidas 1.726 respostas de residentes belo-horizontinos. Essa amostra tem nível de confiança de 99\%, com margem de erro amostral de $5 \%$. A pesquisa abrangeu todas as regionais administrativas de Belo Horizonte, conforme ilustrado na Figura 1. A Regional Centro-Sul foi a que apresentou maior percentual de respondentes (33\%), seguida das regionais Pampulha (15\%) e Oeste (14\%). A Regional Norte, a regional menos populosa da cidade, de acordo com dados da Prefeitura municipal (Belo Horizonte, 2019), obteve apenas 3\% das respostas da amostra.
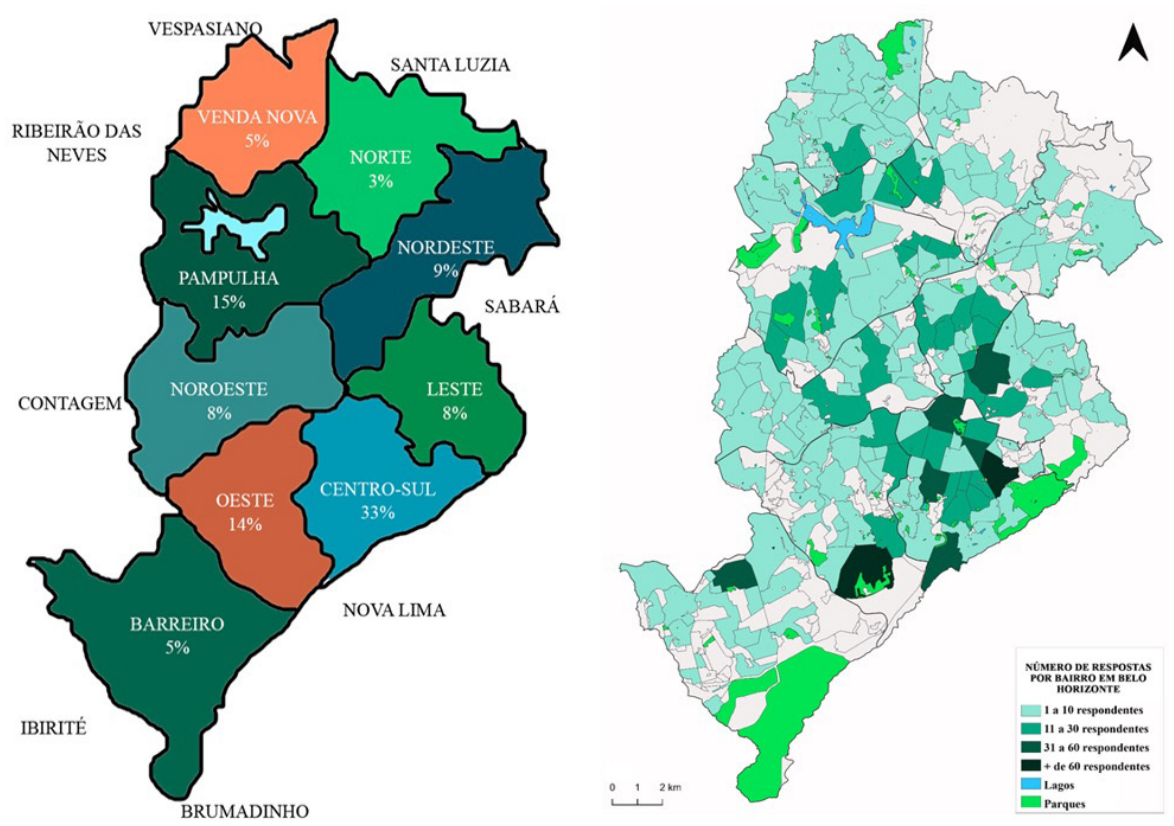

Figura 1. Percentual de respondentes por Regional Administrativa (esquerda) e por bairro (direita) de Belo Horizonte. Fonte: Elaboração própria.

Ainda, apesar de existir maior concentração de respondentes na Regional Centro-Sul, a pesquisa obteve respostas de residentes em 223 barrios de Belo Horizonte, como também ilustrado na Figura 1. Os bairros com maior número de respostas foram: Buritis (4,6\%), na Regional Oeste; Serra (4,4\%), Funcionários (3,2\%) e Santo Antônio 
(2,8\%), na Regional Centro-Sul; e Sagrada Família (2,7\%), na Regional Leste. A amostra utilizada neste estudo permite tirar conclusões válidas para o grupo respondente, mas não permite generalizar as conclusões para todo o território devido à falta de representatividade em alguns bairros da capital.

\section{Caracterização dos respondentes}

O Quadro 3 apresenta a caracterização da amostra, por gênero. A maior parte dos respondentes é do gênero feminino (61\%), convergindo com os dados oficiais, uma vez que $53,8 \%$ da população de Belo Horizonte é constituída por mulheres (Brasil, 2018). Em relação à idade, a maioria dos respondentes (70\%) têm entre 25 e 54 anos, com predominância da faixa etária de 35 a 44 anos (27,8\%) e 25 e 34 anos (24,5\%). Ressalta-se que, entre o público masculino, essas duas faixas etárias apresentam praticamente o mesmo percentual de respostas. Entre as mulheres, $21,1 \%$ tinham acima de 45 anos, diferentemente dos homens, que apresentaram um percentual menor do público mais velho (16,8\%). Em relação à renda familiar, tem-se a predominância de 4 e 10 salários mínimos, com percentuais similares entre os dois gêneros. A maior parte dos respondentes (75,3\%) declarou ter Ensino Superior completo.

Quadro 3. Resumo geral do perfil do respondente por gênero. Fonte: Elaboração própria.

\begin{tabular}{|c|c|c|c|c|c|c|c|}
\hline \multirow[t]{2}{*}{ Categorias } & & \multicolumn{2}{|c|}{ Feminino } & \multicolumn{2}{|c|}{ Masculino } & \multicolumn{2}{|c|}{ Total } \\
\hline & & Total & Percentual & Total & Percentual & Total & Percentual \\
\hline \multirow[t]{6}{*}{ Faixa Etária } & 18-24 anos & 121 & $11,5 \%$ & 62 & $9,2 \%$ & 183 & $10,6 \%$ \\
\hline & $25-34$ anos & 234 & $22,2 \%$ & 189 & $28,1 \%$ & 423 & $24,5 \%$ \\
\hline & $35-44$ anos & 283 & $26,9 \%$ & 197 & $29,3 \%$ & 480 & $27,8 \%$ \\
\hline & $45-54$ anos & 193 & $18,3 \%$ & 112 & $16,6 \%$ & 305 & $17,7 \%$ \\
\hline & $55-64$ anos & 185 & $17,6 \%$ & 83 & $12,3 \%$ & 266 & $15,5 \%$ \\
\hline & 65 ou mais & 37 & $3,5 \%$ & 30 & $4,5 \%$ & 67 & $3,9 \%$ \\
\hline \multirow[t]{6}{*}{ Renda Familiar } & até 1 salário & 17 & $1,6 \%$ & 13 & $1,9 \%$ & 30 & $1,7 \%$ \\
\hline & de 1 a 2 salários & 87 & $8,3 \%$ & 34 & $5,1 \%$ & 121 & $7,0 \%$ \\
\hline & de 2 a 4 salários & 170 & $16,1 \%$ & 101 & $15,0 \%$ & 271 & $15,7 \%$ \\
\hline & de 4 a 10 salários & 371 & $35,2 \%$ & 242 & $36,0 \%$ & 613 & $35,5 \%$ \\
\hline & de 10 a 20 salários & 278 & $26,4 \%$ & 190 & $28,2 \%$ & 468 & $27,1 \%$ \\
\hline & acima de 20 salários & 130 & $12,3 \%$ & 93 & $13,8 \%$ & 223 & $12,9 \%$ \\
\hline \multirow[t]{3}{*}{ Escolaridade } & Ensino Fundamental & 23 & $2,2 \%$ & 24 & $3,6 \%$ & 47 & $2,7 \%$ \\
\hline & Ensino Médio completo & 235 & $22,3 \%$ & 145 & $21,5 \%$ & 380 & $22,0 \%$ \\
\hline & Ensino Superior completo & 795 & $75,5 \%$ & 504 & $74,9 \%$ & 1299 & $75,3 \%$ \\
\hline
\end{tabular}

No Quadro 4 é apresentado o perfil da saúde dos respondentes, por gênero. Os dados indicam que $9,1 \%$ de pessoas fumam regularmente, resultado semelhante ao do Ministério da Saúde do Brasil, que aponta que $11,8 \%$ dos adultos ( $\geq 18$ anos) se declaram fumantes em Belo Horizonte (Brasil, 2018). Conforme a OMS, são consideradas acima do peso as pessoas que apresentam o IMC maior ou igual a $25 \mathrm{~kg} / \mathrm{m}^{2}$. 
Quadro 4. Perfil da amostra sobre a Saúde, por gênero. Fonte: Elaboração própria.

\begin{tabular}{llccc}
\hline \multirow{2}{*}{ Categorias } & & Feminino & Masculino & Total \\
\hline \multirow{2}{*}{ Fumante Regular } & Sim & $8,5 \%$ & $10,0 \%$ & $9,1 \%$ \\
\cline { 2 - 5 } & Não & $91,5 \%$ & $90,0 \%$ & $90,9 \%$ \\
\cline { 2 - 5 } & Abaixo do peso & $3,0 \%$ & $0,9 \%$ & $2,2 \%$ \\
\cline { 2 - 5 } & Peso normal & $56,8 \%$ & $40,4 \%$ & $50,4 \%$ \\
\cline { 2 - 5 } & Sobrepeso & $26,9 \%$ & $41,9 \%$ & $32,7 \%$ \\
\cline { 2 - 5 } & Obesidade I & $8,8 \%$ & $13,4 \%$ & $10,6 \%$ \\
\cline { 2 - 5 } & Obesidade II & $4,1 \%$ & $3,0 \%$ & $3,7 \%$ \\
\cline { 2 - 5 } & Obesidade III & $0,4 \%$ & $0,4 \%$ & $0,4 \%$ \\
\hline \multirow{2}{*}{ Atividade Física } & Fisicamente Ativo & $38,9 \%$ & $51,5 \%$ & $43,8 \%$ \\
\cline { 2 - 5 } & Insuficientemente Ativo & $31,7 \%$ & $28,7 \%$ & $30,5 \%$ \\
\cline { 2 - 5 } & Inativo & $29,4 \%$ & $19,8 \%$ & $25,7 \%$ \\
\hline
\end{tabular}

Os resultados do Quadro 4 também mostram que 47,4\% da amostra possuem sobrepeso ou obesidade e cerca de $14,7 \%$ de pessoas apresentaram obesidade em algum grau. Esses resultados são similares aos dados oficiais, em que 53,3\% dos adultos de Belo Horizonte possuem sobrepeso ou obesidade e 17,2\% dos adultos possuem algum grau de obesidade (Brasil, 2018). Além disso, o percentual de pessoas com excesso de peso atinge mais da metade da população brasileira (Brasil, 2018). Por fim, 56,2\% dos respondentes se declararam insuficientemente ativos ou inativos.

No Quadro 5 é apresentado o perfil da amostra em relação aos deslocamentos diários, por gênero. A maioria dos respondentes (76\%) tem o trabalho como principal motivo de deslocamento. Ainda em relação ao motivo das viagens, importa ressaltar que o motivo das compras se destaca entre as mulheres comparativamente aos homens, com atratividade de $8,5 \%$ e 1,6\%, respectivamente. Tal resultado pode ser explicado em função de mulheres apresentarem, em geral, comportamento diferenciado de suas viagens, em face da realização de múltiplas atividades diárias, as quais superam o simples deslocamento para o trabalho (Cardoso, 2007). O veículo particular motorizado responde pela maioria dos deslocamentos diários entre homens e mulheres, com percentuais superiores a 50\%, no entanto, as mulheres se valem mais dos transportes públicos por ônibus do que o público masculino (27\% e $18,4 \%$, respectivamente), sendo que os diferenciais de renda entre os gêneros podem se afigurar como elemento explicativo deste comportamento. A maioria dos respondentes gasta entre 16 e 30 minutos nesse deslocamento diário principal (38,1\%), percorrendo uma distância média de mais de $7 \mathrm{~km}(41,1 \%)$.

Quadro 5. Perfil da amostra sobre motivo, utilização e tempo de viagens, por gênero. Fonte: Elaboração própria.

\begin{tabular}{llccc}
\hline Categorias & & Feminino & Masculino & Total \\
\hline $\begin{array}{l}\text { Motivo do Deslocamento } \\
\text { Diário }\end{array}$ & Trabalho & $70,5 \%$ & $84,7 \%$ & $76,0 \%$ \\
\cline { 2 - 5 } & Estudo & $13,2 \%$ & $8,6 \%$ & $11,4 \%$ \\
\cline { 2 - 5 } & Compras & $8,5 \%$ & $1,6 \%$ & $5,8 \%$ \\
\cline { 2 - 5 } & Lazer & $3,4 \%$ & $2,7 \%$ & $3,1 \%$ \\
\cline { 2 - 5 } & Outros & $4,5 \%$ & $2,4 \%$ & $3,7 \%$ \\
\hline
\end{tabular}




\begin{tabular}{|c|c|c|c|c|}
\hline Categorias & & Feminino & Masculino & Total \\
\hline \multirow{8}{*}{$\begin{array}{l}\text { Principal Modo de } \\
\text { Transporte }\end{array}$} & Carro & $52,4 \%$ & $51,7 \%$ & $52,1 \%$ \\
\hline & Moto & $1,1 \%$ & $8,0 \%$ & $3,8 \%$ \\
\hline & Ônibus & $27,0 \%$ & $18,4 \%$ & $23,6 \%$ \\
\hline & Metrô & $1,7 \%$ & $1,9 \%$ & $1,8 \%$ \\
\hline & A pé & $10,6 \%$ & $7,9 \%$ & $9,6 \%$ \\
\hline & Bicicleta & $0,9 \%$ & $7,0 \%$ & $3,3 \%$ \\
\hline & Táxi ou serviços & $5,7 \%$ & $4,8 \%$ & $5,3 \%$ \\
\hline & Outros & $0,5 \%$ & $0,3 \%$ & $0,4 \%$ \\
\hline \multirow[t]{5}{*}{ Tempo de Deslocamento } & Até 15 minutos & $24,2 \%$ & $24,4 \%$ & $24,3 \%$ \\
\hline & 16 a 30 minutos & $36,4 \%$ & $40,7 \%$ & $38,1 \%$ \\
\hline & 31 a 1 hora & $27,6 \%$ & $26,4 \%$ & $27,2 \%$ \\
\hline & 1 hora a 2 horas & $10,4 \%$ & $7,9 \%$ & $9,4 \%$ \\
\hline & Mais de 2 horas & $1,3 \%$ & $0,6 \%$ & $1,0 \%$ \\
\hline \multirow{6}{*}{$\begin{array}{l}\text { Distância do } \\
\text { Deslocamento }\end{array}$} & Até $1 \mathrm{~km}$ & $9,7 \%$ & $6,7 \%$ & $6,8 \%$ \\
\hline & Acima de 1 a $2 \mathrm{~km}$ & $12,0 \%$ & $8,6 \%$ & $8,6 \%$ \\
\hline & Acima de 2 a $3 \mathrm{~km}$ & $17,8 \%$ & $15,2 \%$ & $10,6 \%$ \\
\hline & Acima de 3 a $5 \mathrm{~km}$ & $16,0 \%$ & $16,3 \%$ & $16,8 \%$ \\
\hline & Acima de 5 a $7 \mathrm{~km}$ & $7,6 \%$ & $5,6 \%$ & $16,1 \%$ \\
\hline & Mais de 7 km & $36,9 \%$ & $47,6 \%$ & $41,1 \%$ \\
\hline
\end{tabular}

No Quadro 6 é apresentada a percepção dos respondentes em relação à influência dos fatores do ambiente urbano na escolha do transporte ativo (ciclismo ou caminhada). A falta de iluminação das vias, de segurança pública, de segurança viária, velocidade dos veículos nas vias e topografia são aspectos do ambiente urbano que influenciam negativamente a escolha pela caminhada, pois a maioria dos respondentes indicou que esses fatores são um impeditivo ou prejudicam muito os deslocamentos a pé. Em relação aos deslocamentos por bicicleta, a falta de integração com o transporte público, a segurança viária, a segurança pública, a falta de bicicletários e de infraestrutura cicloviária, a velocidade das vias e a topografia são os fatores apontados como impeditivos ou que prejudicam muito. Ainda, em geral, a percepção feminina é mais negativa para os atributos do ambiente urbano considerados. Para escolha da caminhada, por exemplo, fatores ligados à segurança pública ou viária pesam muito mais para as mulheres que para os homens. Na escolha pelo ciclismo, a diferença de percepção urbana se difere mais ainda entre os grupos feminino e masculino, destacando o atributo assédio, em que $43,5 \%$ das mulheres consideram que tal atributo prejudica muito ou é um impeditivo para se pedalar, enquanto apenas $6,9 \%$ dos homens têm esta mesma opinião. Por sua vez, para o atributo topografia, $27,7 \%$ das mulheres acreditam que esta é um impeditivo para o uso da bicicleta, percepção compartilhada por $11,8 \%$ dos homens. 
Quadro 6. Percepção da influência do ambiente urbano no transporte ativo ( $M=$ masculino; $F=$ feminino).

Fonte: Elaboração própria.

\begin{tabular}{|c|c|c|c|c|c|c|c|c|c|}
\hline \multirow[t]{2}{*}{ Modo } & \multirow[t]{2}{*}{ Fatores } & \multicolumn{2}{|c|}{ Impeditivo } & \multicolumn{2}{|c|}{ Prejudica muito } & \multicolumn{2}{|c|}{ Prejudica pouco } & \multicolumn{2}{|c|}{ Não prejudica } \\
\hline & & M & $\mathrm{F}$ & $\mathrm{M}$ & $\mathrm{F}$ & M & $\mathrm{F}$ & M & $\mathrm{F}$ \\
\hline \multirow[t]{10}{*}{ Caminhada } & Iluminação & $14,3 \%$ & $30,4 \%$ & $36,1 \%$ & $44,1 \%$ & $36,0 \%$ & $18,2 \%$ & $13,7 \%$ & $7,3 \%$ \\
\hline & Vestiário & $6,8 \%$ & $4,3 \%$ & $15,5 \%$ & $16,2 \%$ & $26,3 \%$ & $29,0 \%$ & $51,4 \%$ & $50,5 \%$ \\
\hline & Velocidade das vias & $5,5 \%$ & $9,7 \%$ & $30,0 \%$ & $34,6 \%$ & $37,1 \%$ & $38,0 \%$ & $27,3 \%$ & $17,8 \%$ \\
\hline & Segurança Viária & $4,8 \%$ & $11,5 \%$ & $24,5 \%$ & $37,9 \%$ & $39,8 \%$ & $33,0 \%$ & $30,9 \%$ & $17,6 \%$ \\
\hline & Segurança Pública & $15,0 \%$ & $28,6 \%$ & $41,2 \%$ & $50,1 \%$ & $31,4 \%$ & $16,6 \%$ & $12,5 \%$ & $4,7 \%$ \\
\hline & Arborização & $3,7 \%$ & $4,4 \%$ & $29,9 \%$ & $34,5 \%$ & $39,2 \%$ & $42,2 \%$ & $27,2 \%$ & $19,0 \%$ \\
\hline & Ruído & $1,5 \%$ & $1,3 \%$ & $14,9 \%$ & $14,7 \%$ & $34,2 \%$ & $35,2 \%$ & $49,5 \%$ & $48,7 \%$ \\
\hline & Largura das calçadas & $2,2 \%$ & $2,1 \%$ & $22,4 \%$ & $24,9 \%$ & $44,9 \%$ & $46,7 \%$ & $30,5 \%$ & $26,3 \%$ \\
\hline & Condição das calçadas & $2,7 \%$ & $2,8 \%$ & $29,4 \%$ & $36,2 \%$ & $44,6 \%$ & $42,4 \%$ & $23,3 \%$ & $18,6 \%$ \\
\hline & Topografia & $4,3 \%$ & $7,5 \%$ & $21,5 \%$ & $29,9 \%$ & $41,3 \%$ & $40.9 \%$ & $32,8 \%$ & $21,7 \%$ \\
\hline \multirow[t]{9}{*}{ Ciclismo } & Integração transporte público & $15,6 \%$ & $22,2 \%$ & $33,9 \%$ & $39,5 \%$ & $27,2 \%$ & $20,7 \%$ & $23,3 \%$ & $17,7 \%$ \\
\hline & Segurança viária & $18,7 \%$ & $34,1 \%$ & $44,0 \%$ & $49,4 \%$ & $28,6 \%$ & $12,4 \%$ & $8,7 \%$ & $4,2 \%$ \\
\hline & Assédio & $1,6 \%$ & $13,7 \%$ & $5,3 \%$ & $29,8 \%$ & $14,3 \%$ & $31,6 \%$ & $78,8 \%$ & $24,8 \%$ \\
\hline & Segurança Pública & $19,6 \%$ & $33,4 \%$ & $42,8 \%$ & $49,2 \%$ & $28,1 \%$ & $12,7 \%$ & $9,5 \%$ & $4,7 \%$ \\
\hline & Bicicletário & $22,2 \%$ & $27,8 \%$ & $44,3 \%$ & $47,9 \%$ & $24,1 \%$ & $16,5 \%$ & $9,3 \%$ & $7,7 \%$ \\
\hline & Vestiário & $10,4 \%$ & $9,7 \%$ & $26,9 \%$ & $25,1 \%$ & $29,2 \%$ & $27,3 \%$ & $33,4 \%$ & $38,0 \%$ \\
\hline & Infraestrutura cicloviária & $22,2 \%$ & $34,4 \%$ & $50,9 \%$ & $51,3 \%$ & $19,0 \%$ & $10,3 \%$ & $7,9 \%$ & $4,0 \%$ \\
\hline & Velocidade das vias & $13,8 \%$ & $29,6 \%$ & $50,2 \%$ & $52,8 \%$ & $26,7 \%$ & $12,8 \%$ & $9,2 \%$ & $4,8 \%$ \\
\hline & Topografia & $11,8 \%$ & $27,7 \%$ & $39,8 \%$ & $45,3 \%$ & $29.9 \%$ & $18,5 \%$ & $18,5 \%$ & $8,5 \%$ \\
\hline
\end{tabular}

Os resultados indicam uma percepção negativa em relação aos fatores do ambiente urbano na escolha pelos modos ativos. Ainda, observa-se que muitos destes (com exceção da topografia) são passíveis de melhoria para promoção do transporte ativo.

\section{Relação do modo de transporte com o IMC e o sedentarismo}

O Quadro 7 apresenta a relação do modo de transporte, os resultados do IMC e do nível de atividade física. Observa-se que os respondentes que se deslocam a pé ou de bicicleta foram os que apresentaram maior percentual de peso ideal, com percentuais superiores a $60 \%$ nas condições de IMC em questão. Além disso, os usuários do ônibus e metrô que, em geral, utilizam o transporte ativo para acessar o transporte público (especialmente a caminhada), apresentam IMC variando entre o peso ideal e acima do peso.

Quadro 7. Modo de transporte, IMC e Atividade Física (FA: fisicamente ativo; IA: insuficientemente ativo; I: inativo). Fonte: Elaboração própria.

\begin{tabular}{lcccccc}
\hline \multirow{2}{*}{ Modo de transporte } & \multicolumn{1}{c}{ IMC } & \multicolumn{3}{c}{ Atividade Física } \\
& Peso ideal & Acima do peso & Obesidade & FA & IA & I \\
\hline A pé & $64,0 \%$ & $27,0 \%$ & $9,0 \%$ & $50,0 \%$ & $38,0 \%$ & $12,0 \%$ \\
\hline Bicicleta & $70,0 \%$ & $23,0 \%$ & $7,0 \%$ & $86,0 \%$ & $14,0 \%$ & 0 \\
\hline Carro & $51,0 \%$ & $34,0 \%$ & $16,0 \%$ & $45,0 \%$ & $29,0 \%$ & $26,0 \%$ \\
\hline
\end{tabular}




\begin{tabular}{lcccccc}
\hline \multirow{2}{*}{ Modo de transporte } & \multicolumn{1}{c}{ IMC } & \multicolumn{3}{c}{ Atividade Física } \\
& Peso ideal & Acima do peso & Obesidade & FA & IA & I \\
\hline Motocicleta & $41,0 \%$ & $45,0 \%$ & $14,0 \%$ & $59,0 \%$ & $15,0 \%$ & $26,0 \%$ \\
\hline Metrô & $42,0 \%$ & $48,0 \%$ & $10,0 \%$ & $19,0 \%$ & $42,0 \%$ & $39,0 \%$ \\
\hline Ônibus & $52,0 \%$ & $33,0 \%$ & $15,0 \%$ & $35,0 \%$ & $34,0 \%$ & $31,0 \%$ \\
\hline Táxi & $42,0 \%$ & $34,0 \%$ & $24,0 \%$ & $34,0 \%$ & $36,0 \%$ & $30,0 \%$ \\
\hline
\end{tabular}

Os respondentes que se deslocam por carro, motocicleta ou táxi apresentaram maior propensão à obesidade. Analisando o nível de atividade física, observa-se que os usuários da bicicleta são os mais fisicamente ativos, seguido pelos usuários da moto e dos que utilizam a caminhada nos deslocamentos diários. Dessa forma, pode-se supor que o transporte ativo exerce uma influência positiva na redução do sedentarismo.

Ainda, avaliou-se o nível de sedentarismo e o IMC apenas dos respondentes que utilizam o automóvel como principal modo para deslocamento. Os resultados apresentados no Quadro 8 indicam similaridade entre as médias de IMC para os diferentes níveis de atividade física. Entretanto, como o p-valor do Teste ANOVA foi menor que 0,05, existem evidências estatísticas de existir diferença significativa para o IMC entre os respondentes com comportamento sedentário (inativo e insuficientemente ativo) e fisicamente ativo.

Quadro 8. Relação entre o IMC e nível de sedentarismo. Fonte: Elaboração própria.

\begin{tabular}{|c|c|c|c|c|}
\hline Nível de Atividade física & Mínimo & Máximo & Média & Desvio Padrão \\
\hline Inativo & 17,32 & 41,52 & 26,88 & 5,36 \\
\hline Insuficientemente ativo & 16,02 & 39,39 & 25,88 & 4,53 \\
\hline Fisicamente ativo & 15,23 & 38,10 & 24,81 & 3,53 \\
\hline Média Geral & 15,23 & 41,52 & 25,67 & 4,45 \\
\hline $\begin{array}{l}\text { ANOVA } \\
\text { Test- } \mathrm{F}=17,19 \\
\text { p-value }=4,71 \mathrm{E} \text {-o } 8 \\
\text { Comparação de grupos ( } \\
\text { Inativo versus insuficiente } \\
\text { Inativo versus fisicamente } \\
\text { Insuficientemente ativo v }\end{array}$ & $\begin{array}{l}\text { i Test) } \\
\text { ivo: } \mathrm{p} \text {-valor } \\
\text { valor = o,o } \\
\text { amente ati }\end{array}$ & 0,003 & & \\
\hline
\end{tabular}

Complementarmente, foi realizado o teste de comparações múltiplas (Teste de Bonferroni) entre os grupos para identificar as variáveis com médias diferentes, cujos resultados também estão apresentados no Quadro 8. Todas as comparações dois a dois resultaram em um p-valor menor que 0,05 , ou seja, todas as relações entre os grupos são estatisticamente significativas. Assim, pode-se concluir que todos os grupos são diferentes entre si, sendo o grupo inativo com maior índice de IMC, ou seja, os respondentes que utilizam o automóvel mais sedentários apresentam maior média de IMC.

\section{Fatores que influenciam no sedentarismo}

O Quadro 9 apresenta os fatores que influenciam no sedentarismo. Observa-se que o gênero, idade, renda, modo de transporte e o IMC são fatores que influenciam os níveis de sedentarismo, todos com significância estatística ( $\mathrm{p}$-valor $<0,05)$. Enquanto o gênero, a idade e a renda influenciam negativamente (valor do coeficiente estimado), tem-se que o modo de transporte e o IMC influenciam positivamente. Portanto, modos de transporte ativos podem ter uma influência positiva no sedentarismo. 
Quadro 9. Fatores que influenciam no sedentarismo. Fonte: Elaboração própria.

\begin{tabular}{lccccc}
\hline Fatores & Valor estimado & Erro & z-valor & p-valor & Significância \\
\hline Kappa & 1,404 & 0,055 & 25,500 & $<2 \mathrm{e}-16$ & 0,001 \\
\hline Constante & 0,457 & 0,289 & 1,581 & 0,11 & - \\
\hline Gênero & $-0,613$ & 0,096 & $-6,365$ & $1,96 \mathrm{e}-10$ & 0,001 \\
\hline Idade & $-0,077$ & 0,035 & $-2,200$ & 0,02779 & 0,05 \\
\hline Renda & $-0,149$ & 0,041 & $-3,652$ & 0,00026 & 0,001 \\
\hline Modo de Transporte & 0,109 & 0,027 & 4,075 & $4,600-05$ & 0,001 \\
\hline IMC & 0,425 & 0,055 & 7,733 & $1,04 \mathrm{e}-14$ & 0,001 \\
\hline
\end{tabular}

Método de otimização de máxima verossimilhança: Broyden-Fletcher-Goldfarb-Shanno (BFGS)

Log Likelihood: - 1780

Número de observações: 1726

Número de iterações: 48

Considerando os 222 respondentes usuários do transporte ativo (bicicleta e caminhada), os resultados apresentados no Quadro 10 indicam que o modo de transporte influencia negativamente no nível de sedentarismo. Portanto, pode-se dizer que a escolha por modos ativos implica em uma vida menos sedentária, independentemente do gênero, idade e renda familiar.

Quadro 10. Fatores que influenciam no sedentarismo. Fonte: Elaboração própria.

\begin{tabular}{lccccc}
\hline Fatores & Valor estimado & Erro & z-valor & p-valor & Significância \\
\hline Kappa & 2,116 & 0,238 & 8,895 & $<2 \mathrm{e}-16$ & 0,001 \\
\hline Constante & 2,202 & 0,862 & 2,553 & 0,0107 & 0,05 \\
\hline Gênero & $-0,525$ & 0,329 & $-1,598$ & 0,1101 & \\
\hline Idade & $-0,028$ & 0,102 & $-0,278$ & 0,7809 & 0,1 \\
\hline Renda & $-0,202$ & 0,119 & $-1,695$ & 0,0901 & 0,001 \\
\hline Modo de Transporte & $-1,652$ & 0,444 & $-3,719$ & 0,0002 & 0,03 \\
\hline IMC & 0,435 & 0,201 & 2,155 & 0,05 & 0.03 \\
\hline
\end{tabular}

Método de otimização de máxima verossimilhança: Broyden-Fletcher-Goldfarb-Shanno (BFGS)

Log Likelihood: -179,7

Número de observações: 222

Número de iterações: 37

Os resultados apresentados neste artigo sobre a influência para escolha do modo ativo no sedentarismo têm associação com os estudos que abordam o conceito A-S-I (iniciais das palavras evitar, mudar e melhorar em inglês) para se alcançar uma mobilidade urbana sustentável. Nykvist e Whitmarsh (2008) defendem reduzir o uso do automóvel ou reduzir a extensão dos percursos como primeira medida para alcançar uma mobilidade mais sustentável. Uma forma para tornar isto efetivo é por meio da melhoria da oferta e acessibilidade de transporte público.

Além disso, é inegável que uma adequada infraestrutura para pedestres e ciclistas poderia promover uma mobilidade urbana sustentável ao mesmo tempo que a melhoria dos indicadores de saúde dos seus usuários. Dessa forma, uma política pública voltada ao transporte não motorizado pode ter impacto em outros setores, como a saúde. Dado que o ambiente urbano possui características que desestimulam a escolha pela caminhada ou pela bicicleta, a promoção desses fatores é o primeiro caminho para atrair mais usuários para o transporte ativo, provocando uma metamorfose nos indicadores de saúde. 


\section{Conclusões}

Diante dos problemas relacionados à mobilidade urbana e à saúde pública, assim como $o$ alto de índice de sedentarismo, é relevante que sejam analisadas como o ambiente urbano pode contribuir para a utilização do transporte de pessoas. Nesse contexto, este artigo analisou a influência do ambiente urbano na escolha do transporte ativo como forma de deslocamento e suas relações com o sedentarismo das pessoas em Belo Horizonte.

De maneira geral, e de forma descritiva, mulheres têm uma percepção ligeiramente diferente dos homens em relação ao transporte ativo. As mulheres indicaram que alguns aspectos do ambiente urbano, principalmente aqueles ligados à segurança pública $\mathrm{e}$ viária, podem ser um "impeditivo" ou "prejudicar muito" a escolha da caminhada como modo de transporte, quando comparada à percepção dos homens. Em relação ao uso da bicicleta, a (falta de) infraestrutura cicloviária é um impeditivo para homens e mulheres.

Em relação ao IMC, níveis de atividade física e modos de transporte, os resultados deixaram explícito que o transporte ativo reduz o sedentarismo. Ainda, os usuários de modos motorizados têm maiores níveis de obesidade. Por fim, os níveis de obesidade estão relacionados com a falta de atividade física. Dessa forma, políticas de estímulo ao transporte ativo, além de contribuir para a melhoria da mobilidade urbana, podem ajudar na redução do sedentarismo e, consequentemente, da obesidade.

Por fim, os modelos de regressão logística ordenada comprovaram como o transporte ativo influencia positivamente na redução do sedentarismo. Ainda, os modelos indicaram que o IMC tem influência positiva nos níveis de sedentarismo, sugerindo que o incentivo à redução desse índice (perda de peso) tem relevância para incentivar o uso do transporte ativo, já que quanto maiores os níveis de sedentarismo, maior a chance de escolha pelo automóvel. Além disso, a prática de atividade física por meio do deslocamento ativo pode colaborar com a redução do IMC.

Assim, conclui-se que um ambiente urbano atrativo ao ciclismo e à caminhada pode estimular o transporte ativo. Para tanto, a infraestrutura tem papel preponderante na atração de novos usuários para essa modalidade de deslocamento, sendo papel do poder público o investimento em infraestrutura compatível com os objetivos para alcançar uma mobilidade urbana sustentável e de qualidade nas cidades brasileiras.

Como futuros trabalhos, sugere-se a ampliação desta pesquisa para outras cidades, com diferentes características urbanas para comparação dos resultados e obtenção de conclusões generalizáveis. 


\section{Q Bibliografia}

» ABESO (2016). Diretrizes Brasileiras de Obesidade. ABESO: São Paulo.

"Anable, J. e Gatersleben, B. (2005). All work and no play? The role of instrumental and affective factors in work and leisure journeys by different travel modes. Transportation Research Part A, v. 39, n. 2-3, p. 163-181.

»Bell, A. C.; Keyou, G. E. e Popkin, B. M. (2002). The road to obesity or the path to prevention: motorized transportation and obesity in China. Obesity Research, v. 10, n. 4, p. 277-283.

» BELO HORIZONTE. PRODABEL. Regionais Administrativas de Belo Horizonte. 2019. Disponível em: https://prefeitura.pbh.gov.br/noticias/prodabel-detalhatamanho-e-numero-de-bairros-das-regionais. Acesso em: 21 de janeiro de 2019.

»Brasil (2012). Política Nacional de Mobilidade Urbana. Ministério das Cidades: Brasília.

»Brasil (2017). Saúde Suplementar: vigilância de fatores de risco e proteção para doenças crônicas por inquérito telefônico. Ministério da Saúde: Brasília.

"Brasil (2018). Panorama Belo Horizonte. IBGE.

"Cardoso, L. (2007). Transporte público, acessibilidade urbana e desigualdades socioespaciais na região metropolitana de Belo Horizonte. Tese (Doutorado em Geografia). Instituto de Geociências. Universidade Federal de Minas Gerais.

» CNT e NTU (2017). Pesquisa mobilidade da população urbana. CNT e NTU: Brasília.

»Gehl, J. e Svarre, B. B. (2017). A dimensão humana: uma abordagem sustentável do planejamento urbano. In: ANDRADE, V. e LINKE, C. C (Orgs). Cidade de Pedestres: a caminhabilidade no Brasil e no mundo. Rio de Janeiro: Babilonia Cultura Editorial, 2017. Cap. 1, p. 13-18.

» Giles-Corti, B.; Vernez-Moudon, A.; Reis, R.; Turell, G.; Dannemberg, A. L.; Badland, H.; Foster, S.; Lowe, M.; Sallis, J. F.; Stevenson, M. e Owen, N. (2016). City planning and population health: a global challenge. Urban Design, Transport, and Health, v. 388, n. 10062, p. 2912-2924.

»González-Gross, M. e Meléndez, A. (2013). Sedentarism, active lifestyle and sport: Impact on health and obesity prevention. Nutrición Hospitalaria, v. 28, n. 5, p. 89-98.

"Lajeunesse, S. e Rodríguez, D. A. (2012). Mindfulness, time affluence, and journey-based affect: exploring relationships. Transportation Research Part F: traffic psychology and behaviour, v. 15, n. 2, p. 196-205.

» Machado, L. (2010). Índice de Mobilidade Sustentável para Avaliar a Qualidade de Vida Urbana. Estudo de Caso: Região Metropolitana de Porto Alegre RMPA. Universidade Federal do Rio Grande do Sul.

» Madeira, M. C.; Siqueira, F. C. V.; Facchini, L. A.; Silveira, D. S.; Tomasi, E.; Thumé, E.; Silva, S. M.; Dilélio, A. e Piccinni, R. X. (2013). Atividade física no deslocamento em adultos e idosos do Brasil: prevalências e fatores associados. Cadernos de Saúde Pública, v. 29, p. 165-174. 
» Matos, S. M. A.; Pitanga, F. J. G.; Almeida, M. D. C. C.; Queiroz, C. O.; Santos, C. A.; Almeida, R. T.; Silva, I. T. M.; Griep, R. H.; Amorim, L. D. A. F.; Patrão, A. L. e Aquino, E. M. L. (2018). What Factors Explain Bicycling and Walking for Commuting by ELSA-Brasil Participants? American Journal of Health Promotion, v. 32, n. 3, p. 646-656.

» Nykvist, B. e Whitmarsh, L. (2008). A multi-level analysis of sustainable mobility transitions: Niche development in the UK and Sweden. Technological forecasting and social change, 2008, p. 1373-1387.

» Reynolds, C.O.; Winters, M.; Ries, F. J. e Gouge, B. (2010) Active transportation in urban areas: exploring health benefits and risks. National Collaboration Centre for Environmental Health, Report.

» Sarrias, M. (2016). Discrete Choice Models with Random Parameters in R: The Rchoice Package. Journal of Statistics Software, v.74, n.10, p. 1-31.

» Scheepers, C. E.; Wendel-Vos, G. C. W.; Broederb, J. M.; Van Kempen, E. E. M. M.; Van Wesemael, P. J. V. e Schuit, A. J. (2014). Shifting from car to active transport: a systematic review of the effectiveness of interventions. Transportation Research Part A, v. 70, p. 264-28o.

»Silva, P. T. (2015). Qualidade de Vida Urbana e Mobilidade Urbana Sustentável na Cidade do Porto - Elaboração de um conjunto de indicadores. Universidade do Porto.

» St-Louis, E.; Manaugh, K.; Lierop, D. e El-Genedya, A. (2014). The happy commuter: A comparison of commuter satisfaction across modes. Transportation Research Part F, v. 26, p. 160-170.

»Vieira, R.; Packer, G. Z. e Meneses, R. N. (2016). Índice de caminhabilidade de Blumenau em Santa Catarina/Brasil: uma análise do Centro e do bairro Badenfurt. Anais do VIII Seminario Internacional de Investigación en Urbanismo, Barcelona.

»Villanueva, K.; Badland, H.; Hooper, P.; Koohsari, M. J.; Mavoa, S.; Davern, M.; Roberts, R.; Goldfeld, S. e Giles-Corti, B. (2015). Developing indicators of public open space to promote health and wellbeing in communities. Applied Geography, v. 57, p. 112-119.

"Washington (2018). Physical Activity Guidelines Advisory Committee. Physical Activity Guidelines Advisory Committee Scientific Report. U.S. Department of Health and Human Services: Washington, DC.

"WHO (2006). Obesity and overweight and what is the scale of the obesity problem in your country. Report of a WHO consultation on obesity. Geneva.

»WHO (2017). Health economic assessment tool (HEAT) for walking and for cycling: Methods and user guide on physical activity, air pollution, injuries and carbon impact assessments. Geneva.

\section{Janaina Amorim Dias / janaina.mobilidade@gmail.com}

Arquiteta Urbanista, mestre em Engenharia de Transportes pela UFMG (2020). Sóciodiretora da empresa Metrics Mobilidade. Trabalha há 16 anos com Mobilidade Urbana, especialmente na área de Projetos Viários e Intervenções Urbanas com foco na Mobilidade Ativa. 


\section{Leandro Cardoso / leandro@etg.ufmg.br}

Geógrafo, Mestre e Doutor em Geografia. Professor da Universidade Federal de Minas Gerais, atua nas áreas de Geografia e Engenharia de Transportes, com pesquisas focadas em Planejamento dos Sistemas de Transporte, Mobilidade Urbana Sustentável e Desigualdades Socioespaciais.

\section{Leise Kelli de Oliveira / leise@etg.ufmg.br}

Matemática. Mestre e Doutora em Engenharia de Produção. Pesquisadora de Produtividade do CNPq. Investiga questões relacionadas ao transporte urbano de carga e na aplicabilidade de modelagem matemática no planejamento de transportes.

\section{Bárbara Abreu Matos / barbara.matos@ufop.edu.br}

Engenheira Civil, Mestre em Transportes e Doutoranda em Geografia (Universidade Federal de Minas Gerais). Professora do Departamento de Engenharia Urbana da Universidade Federal de Ouro Preto (DEURB/UFOP), atuando na área de Planejamento e Organização do Sistema de Transporte.

\section{Daniela Antunes Lessa / daniela.lessa@ufop.edu.br}

Engenheira Civil. Mestre em Transportes e Doutora em Geografia. Atua nas linhas de pesquisa de Mobilidade/Acessibilidade espacial da população, Organização do Espaço e Geografia dos Transportes, incluindo a utilização de métodos quantitativos aplicados à análise espacial.

\section{Marconi Gomes da Silva / sportifmarconi@gmail.com}

Cardiologista e Médico do Esporte. Membro diretor da Sociedade Mineira de Cardiologia - SMC e Sociedade Mineira de Medicina do Exercício e do Esporte - SMEXE. Sócio diretor da SPORTIF - Clínica do Exercício e do Esporte. Presidente do grupo de estudos em cardiologia do esporte do Departamento de Ergometria, Medicina Nuclear e Reabilitação Cardiopulmonar - DERC - SBC. 